\title{
Heat and Mass Transfer Flow Past an Infinite Vertical Plate with Variable Thermal Conductivity
}

\author{
I. J. Uwanta \\ Department of Mathematics, \\ Usmanu Danfodiyo University, \\ Sokoto-Nigeria
}

\author{
Murtala Sani \\ Department of Mathematics \& \\ Computer Science, \\ Umaru Musa Yar'adua \\ University, Kasina-Nigeria
}

\begin{abstract}
This investigation is undertaken to study the heat and mass transfer flow past an infinite vertical plate with variable thermal conductivity. The governing equations for the model are formulated with appropriate boundary conditions. The equations are simplified, non-dimensionalized and then solved numerically with the aid of MAPLE package. The flow phenomenon are characterized by the flow parameters such as Prandtl number (Pr), Schmidt number (Sc), Eckert number (Ec), magnetic field $(\mathrm{M})$, porosity $(\mathrm{K})$, thermal Grashof number $(\mathrm{Gr})$, mass Grashof number $(\mathrm{Gc})$, radiation $(\mathrm{N})$, suction $(\alpha)$, thermal conductivity $(\tau)$, chemical reaction $(\mathrm{Kr})$ and reaction order (n) which are studied for velocity field, temperature field and concentration distribution presented graphically.
\end{abstract}

\section{Keywords}

Heat Transfer, Mass Transfer, Thermal Conductivity, Vertical Plate.

\section{INTRODUCTION}

A lot of authors studied heat and mass transfer in nonNewtonian fluid of different type, most especially in power law and higher order fluids. Recently, various researchers have shown interests in the study of non-Newtonian fluids due to its importance in industrial processes. The development of the theory of non- Newtonian fluid mechanics arose from the inadequacy of the theory of Newtonian fluids in predicting the behaviours of many fluids especially those of high molecular weight. Muthucumaraswamy and Senthil Kumar [1] investigated the heat and mass transfer effects on moving vertical plate in the presence of thermal radiation. Gupta et al. [2] have studied Heat and mass transfer on a stretching sheet with suction or blowing. Sattar [3] has discussed the free convection and mass transfer flow through a porous medium past an infinite vertical porous plate with time dependent temperature and concentration. Israel-Cookey et al. [4] have studied the influence of viscous dissipation and radiation on unsteady MHD free convection flow past an infinite heated vertical plate in a porous medium with time dependent suction.

Free convection flow is a significant factor in several practical applications that include, for example, cooling of electronic components, in designs related to thermal insulation, material processing, and geothermal systems, etc. Transient natural convection is of fundamental interest in many industrial and environmental situations such as air conditioning systems, atmospheric flows, motors, thermal regulation process, cooling of electronic devices, and security of energy systems. Illingworth [5] was the first to study the transient free convection flow past an infinite vertical isothermal plate by assuming the Prandtl number as unity. It was Gebhart [6] who first showed that in steady free convection flow, the viscous dissipation heat cannot be neglected for fluids with high Prandtl number or flow at high gravitational field or in rotational flow. Hence, it is essential to know the effects of viscous dissipative heat on the transient free convective flow past an infinite vertical plate with a step-change in temperature. So Soundalgekar et al. [7] studied the effects of viscous dissipative heat on the transient free convection flow past an infinite vertical plate with a step change in plate temperature. The problem governed by nonlinear coupled partial differential equations was solved by finite difference method.

Buoyancy is also of importance in an environment where differences between land and air temperatures can give rise to complicated flow patterns. Das et al. [8] have analyzed radiation effects on flow past an impulsively started infinite isothermal vertical plate. Combined buoyancy-generated heat and mass transfer, due to temperature and concentration variations in fluid saturated porous medium have several important applications in variety of engineering processes including heat exchanger devices, petroleum reservoirs, chemical catalytic reactors, solar energy porous wafer collector systems, ceramic materials, migration of moisture through air contained in fibrous insulations and grain storage installations, and the dispersion of chemical contaminants through water saturated soil, super convecting geothermics, Riley et al. [9]. The buoyancy force induced by density differences in a fluid cause's natural convection. Natural convection flows are frequently encountered in physics and engineering problems such as chemical catalytic reactors, nuclear waste material, etc. Transient free convection is important in many practical applications such as thermal regulation process, security of energy systems, etc. When a conductive fluid moves through a magnetic field, an ionized gas is electrically conductive and the fluid may be influenced by the magnetic field. Magnetohydrodynamic free convection heat transfer flow has considerable interest in the technical field due to its frequent occurrence in industrial technology and geothermal applications, liquid metal fluid and MHD power generation systems, etc. Transport processes in porous media are encountered in a broad range of scientific and engineering problems associated with the fiber and granular insulation materials, packed-bed chemical reactors and transpiration cooling. The change in wall temperature causing 
the free convection flow could be a sudden or a periodic one, leading to a variation in the flow. The vertical free convection boundary layer flow in porous media owing to combined heat and mass transfer has been investigated by Nield [10]. Kishan and Srinivas [11] investigated the effects of thermophores and viscous dissipation on MHD mixed convection, heat and mass transfer about an isothermal vertical flat plate embedded in a fluid saturated porous media. Ramachandra and Bhaskar [12] studied radiation and mass transfer effects on an unsteady MHD free convection flow past a semi-infinite vertical permeable moving plate embedded in a porous media with viscous dissipation. They solved the problem by using the regular perturbation method. Magnetohydrodynamic has attracted the attention of a large number of scholars due to its diverse applications. In astrophysics and geophysics, it is applied to study the stellar and solar structures, interstellar matter, radio propagation through the ionosphere, Riley et al. [9]. In engineering, it finds applications in MHD pumps, MHD bearings etc. Convection in porous media has applications in geothermal energy recovery, oil extraction, thermal energy storage and flow through filtering devices. Convective heat transfer in porous media has received considerable attention in recent years owing to its importance in various technological applications such as fibre and granular insulation, electronic system cooling, cool combustors, and porous material regenerative heat exchangers.

In the processes involving high temperatures, the radiation heat transfer in combination with conduction, convection and also mass transfer plays very important role in the design of pertinent equipments in the areas such as nuclear power plants, gas turbines and the various propulsion devices for aircrafts, missiles, satellites and space vehicles. Moreover, radiation effect is significant in the dynamics of fluid in chemical, environmental, mechanical and solar power engineering. Thus, Chamkha [13] studied the effects of solar radiation on free convection in an isotropic, uniform porous medium supported by a vertical flat plate using a computational method. Also, Sangapatnam et al. [14] considered the radiation and mass transfer effects on MHD free convection flow past impulsively-started isothermal vertical plate with dissipation. The role of thermal radiation is significant in some industrial applications such as glass production and furnace design and in space technology applications, such as cosmical flight aerodynamics rocket, propulsion systems, plasma physics and space craft reentry aerothermodynamics which operate at high temperatures, Riley et al. [9]. Viscous mechanical dissipation effects are very important in geophysical flows and also in certain industrial operations and are usually characterized by the Eckert number. Yasar and Moses [15] developed a onedimensional adaptive-grid finite-differencing computer code for thermal radiation magneto hydro dynamic (RMHD) simulations of fusion plasmas. Aboeldahab and Azzam [16] described the effects of magnetic field on hydro magnetic mixed free-forced heat and mass convection of a gray, optically-thick, electrically conducting viscous fluid along a semi-infinite inclined plate for high temperature and concentration using the Rosseland approximation. Alagoa et al. [17] studied magneto hydro dynamic optically-transparent free convection flow, with radiative heat transfer in porous media with time-dependent suction using an asymptotic approximation, showing that thermal radiation exerts a significant effect on the flow dynamics. Israel-Cookey et al. [4] described the effects of viscous dissipation and thermal radiation on transient magneto hydro dynamic free convection flow past an infinite vertical heated plate in an optically thin environment with time dependent suction showing that increased cooling (positive Grashof number) of the plate and increasing Eckert number boost velocity profile and temperature, a rise in magnetic field, thermal radiation and Darcian drag force decelerate the flow and increasing thermal radiation and magnetic field cool the flow in the porous medium. Nield and Bejan [18], Bejan and Kraus [19] and Ingham et al. [20] excellently describe the extent of the research information in this area. Recently Suneetha et al. [21] studied the effects of thermal radiation on the natural conductive heat and mass transfer of a viscous incompressible gray absorbing-emitting fluid flowing past an impulsively started moving vertical plate with viscous dissipation. An exact solution to the flow of a viscous incompressible unsteady flow past an infinite vertical oscillating plate with variable temperature and mass diffusion by taking into account the homogeneous chemical reaction of first order was reported by Muthucumaraswamy et al. [22].

In nuclear engineering, cooling of medium is more important from the safety point of view and during this cooling process the plate temperature starts oscillating about a non-zero constant mean temperature. The oscillatory flow has applications in industrial and aerospace engineering.

Although different authors studied heat and mass transfer with or without radiation and viscous dissipation effects on the flow past an infinite vertical plate by considering different surface conditions but the study on the effects of radiation on free convection heat and mass transfer with variable thermal conductivity, viscous dissipation and reaction order on flow past an infinite vertical plate has not been found in the literature and hence the motivation to undertake this study. It is therefore proposed to study the heat and mass transfer flow past an infinite vertical plate with variable thermal conductivity.

\section{PROBLEM FORMULATION}

We consider an unsteady infinite vertical isothermal porous plate of laminar natural convection flow of dissipative and radiating fluid in the presence of transverse magnetic field surrounded on one side by infinite mass of fluid like air or water and both at same temperature $T_{\infty}^{\prime}$ and the mass concentration $C_{\infty}^{\prime}$ initially. At time $t^{\prime}>0$, the plate temperature and the mass concentration is raised to $T_{w}^{\prime}$ and $C_{w}^{\prime}$, causing the presence of temperature and concentration difference $T_{w}^{\prime}-T_{\infty}^{\prime}$ and $C_{w}^{\prime}-C_{\infty}^{\prime}$ respectively. As the plate is infinite in extent, the physical variables are functions of $\mathrm{y}^{\prime}$ and $\mathrm{t}^{\prime}$ where $\mathrm{y}^{\prime}$ is taken normal to the plate and the $\mathrm{x}^{\prime}$-axis is taken along the plate in the vertically upward direction. Under the Boussinesq approximation, the governing equations in this case Sharma (2005) and Sahin (2010) for the flow are continuity, momentum, mass concentration and energy respectively are

$\frac{\partial v^{\prime}}{\partial y^{\prime}}=0$ 


$$
\begin{aligned}
\frac{\partial u^{\prime}}{\partial t^{\prime}}+v^{\prime} \frac{\partial u^{\prime}}{\partial y^{\prime}}= & v \frac{\partial^{2} u^{\prime}}{\partial y^{\prime 2}}-\frac{\sigma B_{0}^{2} u^{\prime}}{\rho}-\frac{v u^{\prime}}{K^{*}}+g \beta\left(T^{\prime}-T_{\infty}^{\prime}\right) \\
& +g \beta^{*}\left(C^{\prime}-C_{\infty}^{\prime}\right) \\
\frac{\partial C^{\prime}}{\partial t^{\prime}}+v^{\prime} \frac{\partial C^{\prime}}{\partial y^{\prime}}= & D \frac{\partial^{2} C^{\prime}}{\partial y^{\prime 2}}-R^{*}\left(C^{\prime}-C^{\prime}\right)^{n} \\
\frac{\partial T^{\prime}}{\partial t^{\prime}}+v^{\prime} \frac{\partial T^{\prime}}{\partial y^{\prime}}= & \frac{1}{\rho C_{\rho}} \frac{\partial}{\partial y^{\prime}}\left(K(T) \frac{\partial T^{\prime}}{\partial y^{\prime}}\right)-\frac{1}{\rho C_{\rho}} \frac{\partial q_{r}}{\partial y^{\prime}} \\
& +\frac{v}{\mathrm{C}_{\rho}}\left(\frac{\partial u^{\prime}}{\partial y^{\prime}}\right)^{2}
\end{aligned}
$$

The initial and boundary conditions relevant to the fluid flow are:

$$
\begin{array}{ll}
t^{\prime} \leq 0, & u^{\prime}=0, T^{\prime} \rightarrow T_{\infty}^{\prime}, C^{\prime} \rightarrow C_{\infty}^{\prime} \quad \text { for all } y^{\prime} \\
t^{\prime}>0, & u^{\prime}=0, T^{\prime}=T_{w}^{\prime}, C^{\prime}=C_{w}^{\prime} \quad \text { at } y^{\prime}=0 \\
& u^{\prime} \rightarrow 0, T^{\prime} \rightarrow T_{\infty}^{\prime}, C^{\prime} \rightarrow C_{\infty}^{\prime} \text { as } y^{\prime} \rightarrow \infty
\end{array}
$$

where $U$ is the kinematic viscosity of the grey fluid, $\sigma$ is the Stefan-Boltzmann constant, $B_{0}$ is the constant magnetic field intensity, $\rho$ is density, $K^{*}$ is the permeability, $g$ is the gravitational constant, $\beta$ is the thermal expansion coefficient, $\beta^{*}$ is the concentration expansion coefficient, $T^{\prime}$ is the temperature, $c^{\prime}$ is the mass concentration, $\mathrm{D}$ is the chemical molecular diffusivity, $R^{*}$ is the chemical reaction, $k_{0}$ is the mean absorption coefficient of thermal expansion, $C_{\rho}$ is heat capacity at constant pressure, $q_{r}$ is the radiative heat flux, $u^{\prime}$ and $v^{\prime}$ are velocity components in $x$ and $y$ directions respectively, $t$ is the time, $\mathrm{K}(\mathrm{T})$ is the thermal conductivity, $T_{w}^{\prime}$ is the wall temperature, $T_{\infty}^{\prime}$ is the free stream temperature, $C_{w}^{\prime}$ is the species concentration at the plate surface, $C_{\infty}^{\prime}$ is the free stream concentration.

Assuming the radiative heat flux from the Rosseland approximation to have the form

$$
\frac{\partial q_{r}}{\partial y^{\prime}}=-4 \sigma a^{*}\left(T_{\infty}^{\prime 4}-T^{\prime 4}\right)
$$

$\sigma$ is the Stefan-Boltzmann constant, $a^{*}$ is the mean absorption effect for thermal radiation constant. We assume that the temperature differences within the flow are sufficiently small such that $T^{\prime 4}$ can be expanded in a Taylor series about $T_{\infty}^{\prime}$ and neglecting higher order terms give

$$
T^{\prime 4} \approx 4 T^{\prime} T_{\infty}^{\prime 3}-3 T_{\infty}^{\prime 4}
$$

The thermal conductivity depends on temperature. It is used by Molla et al. (2005), as follows

$$
K(T)=k_{0}\left\{1+\gamma\left(T^{\prime}-T_{\infty}^{\prime}\right)\right\}
$$

where $k_{0}$ is the thermal conductivity of the ambient fluid and $\gamma$ is defined by $\gamma=\frac{1}{K(T)}\left(\frac{\partial K(T)}{\partial T}\right)$

\section{ANALYTICAL SOLUTION}

To solve the governing equations in dimensionless form, we introduce the following non-dimensional quantities:

$$
\begin{aligned}
& U=\frac{u^{\prime}}{U_{0}}, y=\frac{y^{\prime} U_{0}}{v}, t=\frac{t^{\prime} U_{0}^{2}}{v}, \theta=\frac{T^{\prime}-T_{\infty}^{\prime}}{T_{w}^{\prime}-T_{\infty}^{\prime}}, \\
& C=\frac{C^{\prime}-C_{\infty}^{\prime}}{C_{w}^{\prime}-C_{\infty}^{\prime}}, \alpha=\frac{v_{0}}{U_{0}}, \tau=\gamma\left(T_{w}^{\prime}-T_{\infty}^{\prime}\right), \\
& S c=\frac{v}{D}, \operatorname{Pr}=\frac{v \rho c_{\rho}}{k_{0}}, E c=\frac{U_{0}^{2}}{c_{\rho}\left(T_{w}^{\prime}-T_{\infty}^{\prime}\right)}, \\
& G r=\frac{g \beta v\left(T_{w}^{\prime}-T_{\infty}^{\prime}\right)}{U_{0}^{3}}, G c=\frac{g \beta^{*} v\left(C_{w}^{\prime}-C_{\infty}^{\prime}\right)}{U_{0}^{3}}, \\
& K=\frac{K^{*} U_{0}^{2}}{v}, N=\frac{16 a \sigma^{*} T_{\infty}^{\prime 3} v}{k_{0} U_{0}^{2}}, M=\frac{v \sigma B_{0}^{2}}{\rho U_{0}^{2}}, \\
& K r=\frac{v R^{*}\left(C_{w}^{\prime}-C_{\infty}^{\prime}\right)^{n-1}}{U_{0}^{2}},
\end{aligned}
$$

The governing equations on using (9) into (1), (2), (3), (5) and using (6) to (9) into (4) reduce to the following

$$
\begin{aligned}
& \frac{\partial U}{\partial t}-\alpha \frac{\partial U}{\partial y}=\frac{\partial^{2} U}{\partial y^{2}}-M U-\frac{1}{K} U+G r \theta+G c C \\
& \frac{\partial C}{\partial t}-\alpha \frac{\partial C}{\partial y}=\frac{1}{S c} \frac{\partial^{2} C}{\partial y^{2}}-K r C^{n} \\
& \frac{\partial \theta}{\partial t}-\alpha \frac{\partial \theta}{\partial y}=\frac{(1+\tau \theta)}{\operatorname{Pr}} \frac{\partial^{2} \theta}{\partial y^{2}}+\frac{\tau}{\operatorname{Pr}}\left(\frac{\partial \theta}{\partial y}\right)^{2}-\frac{N}{\operatorname{Pr}} \theta \\
& +E c\left(\frac{\partial U}{\partial y}\right)^{2}
\end{aligned}
$$

subject to the boundary conditions

$$
\begin{array}{lll}
t \leq 0, & U=0, \theta \rightarrow 0, C \rightarrow 0 & \text { for all } y \\
t>0, & U=0, \theta=1, C=1 & \text { at } y=0 \\
& U \rightarrow 0, \theta \rightarrow 0, C \rightarrow 0 & \text { as } y \rightarrow \infty
\end{array}
$$


where Pr is the Prandtl number, Sc is Schmidt number, Ec is Eckert number, Gr is thermal Grashof number, Gc is mass Grashof number, and $\mathrm{M}$ is magnetic field, $\mathrm{K}$ is porosity, $\mathrm{N}$ is the radiation, $\alpha$ is suction, $\tau$ is the thermal conductivity, $\mathrm{Kr}$ is the chemical reaction and $\mathrm{n}$ is reaction order parameters.

\section{NUMERICAL PROCEDURE}

The unsteady coupled non-linear partial differential equations (10) - (12) with the boundary conditions (13) were solved using the implicit finite difference scheme of the CrankNicolson type. This method has been extensively developed in recent years and remains one of the best reliable methods for solving partial differential equations. The partial differential equations are converted to difference equation. The CrankNicolson method converges fast and is unconditionally stable.

\section{RESULTS AND DISCUSSIONS}

The numerical solutions are simulated for different values of the Prandtl number Pr, Schmidt number Sc, Eckert number Ec, thermal Grashof number Gr, mass Grashof number Gc, radiation parameter $\mathrm{N}$, magnetic field parameter $\mathrm{M}$, porosity parameter $\mathrm{K}$, thermal conductivity parameter $\tau$, suction parameter $\alpha$, reaction order parameter $\mathrm{n}$ and chemical reaction parameter $\mathrm{Kr}$. The following parameters values are fixed throughout the calculations except where otherwise stated, $\operatorname{Pr}=0.71, \mathrm{Sc}=0.62, \mathrm{Ec}=0.01, \mathrm{M}=5.0, \mathrm{~K}=1.0, \mathrm{Gr}=$ $0.1, \mathrm{Gc}=0.1, \mathrm{~N}=1.0, \alpha=0.2, \tau=1.0, \mathrm{Kr}=0.5, \mathrm{n}=1.0, \mathrm{t}$ $=0.5, \mathrm{y}=0 . .3 .5$.

The velocity profiles are illustrated in Figures 1 to 11 for different values of Prandtl number $(\operatorname{Pr}=0.22,0.71,1.0,7.0)$, Schmidt number $(\mathrm{Sc}=0.22,0.62,0.78,2.63)$, magnetic field parameter $(\mathrm{M}=0.5,1.0,1.5,2.0)$, porosity parameter $(\mathrm{K}=$ $0.1,0.25,0.5,0.75)$, thermal Grashof number $(\mathrm{Gr}=0.1,0.5$, $1.0,1.5)$, mass Grashof number $(\mathrm{Gc}=0.1,0.5,1.0,1.5)$, radiation parameter $(\mathrm{N}=1,5,10,15)$, suction parameter ( $\alpha=0.1,0.5,1.0,1.5)$, thermal conductivity parameter ( $\tau=$ $0.1,0.5,1.0,1.5)$, chemical reaction parameter $(\mathrm{Kr}=1,2,3$, $4)$ and time $(t=0.1,0.2,0.3,0.4)$.

In Figure 1, it is seen that the velocity decrease with increasing Prandtl number and likewise Figure 2 reveals that, the velocity decrease with increase in the Schmidt number. While, Figure 3 shows that, the velocity decreases with increase in the magnetic field parameter. Similarly, Figure 4 indicates that, the velocity increases whenever porosity parameter increases. Also, Figure 5 represents the thermal Grashof number and it is observed that, the velocity increase with increasing thermal Grashof number just like Figure 6 which reveals that, the velocity increase when the mass Grashof number increased. From Figure 7, it shows that, the velocity decrease with increasing radiation parameter and similarly for the suction parameter, Figure 8 indicates that, the velocity decrease with increase in the suction parameter. In Figure 9, it is seen that, the velocity increase with increasing thermal conductivity parameter whereas Figure 10 found that, the velocity decrease when the chemical reaction parameter increase. Figure 11 indicates that, the velocity increase whenever the time increased.

The temperature profiles have been studied and presented in Figures 12 to 16 for different values of Prandtl number $(\mathrm{Pr}=$ $0.71,0.85,1.0,7.0)$, radiation parameter $(\mathrm{N}=1,5,10,15)$, suction parameter ( $\alpha=1,2,3,4)$, thermal conductivity parameter $(\tau=0.1,0.5,1.0,1.5)$ and time $(\mathrm{t}=0.05,0.1,0.2$, 30) shown in Figures $12,13,14,15$ and 16 respectively. In
Figure 12, it is observed that, the temperature decrease with increase in the Prandtl number. Also, Figure 13 reveals that the temperature decrease with increase of the radiation parameter and similarly for the suction parameter, Figure 14 indicates that, the temperature decrease whenever the suction parameter increased. Figure 15 shows that, the temperature increase with increase of the thermal conductivity parameter. Similarly, Figure 16 found that, the temperature increase with respect to increase in time.

The concentration profiles are illustrated in Figures 17 to 21 for different values of Schmidt number $(\mathrm{Sc}=0.22,0.62,0.78$, 2.63 ), suction parameter ( $\alpha=1,2,3,4)$, chemical reaction parameter $(\mathrm{Kr}=0.1,1.0,2.0,3.0)$, reaction order parameter $(\mathrm{n}$ $=1,2,3,4)$ and time $(\mathrm{t}=0.05,0.1,0.2,0.3)$ as in Figures 17, $18,19,20$ and 21 respectively. In Figure 17, it is noticed that, the concentration decrease with increase in the Schmidt number and also Figure 18 reveals that, the concentration decrease with increase of the suction parameter. Similarly, Figure 19 indicates that, the concentration decrease whenever the chemical reaction parameter increased. But, it is observed from Figure 20 that, the concentration increases as the reaction order parameter increase and likewise increase in time increase the concentration as shown in Figure 21.

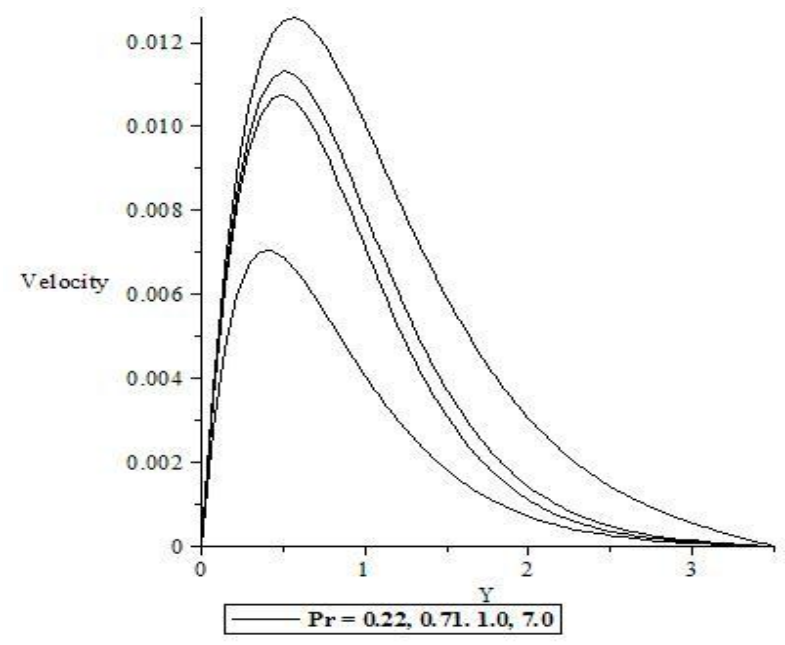

Figure 1: Variation of Velocity against y for different values of Pr.

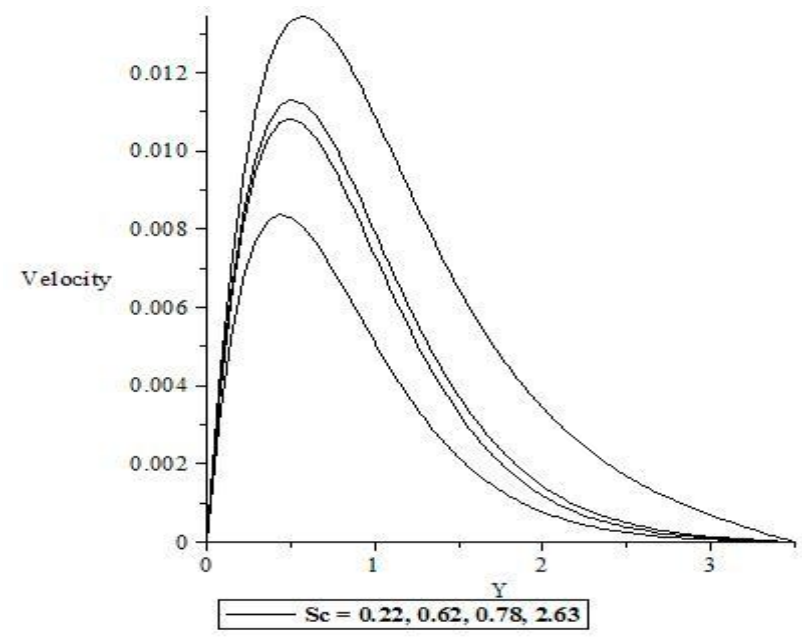


Figure 2: Variation of Velocity against y for different values of Sc.

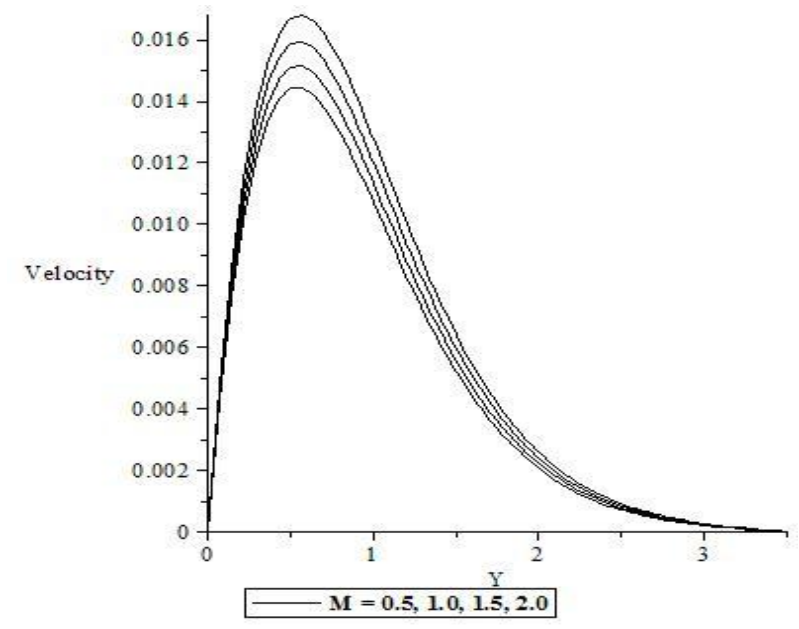

Figure 3: Variation of Velocity against y for different values of $\mathrm{M}$.

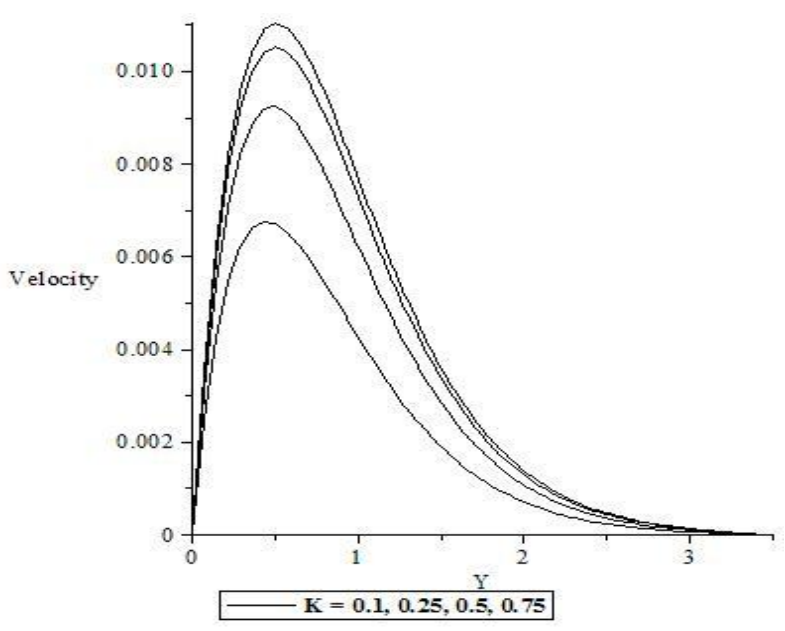

Figure 4: Variation of Velocity against y for different values of $\mathrm{K}$.

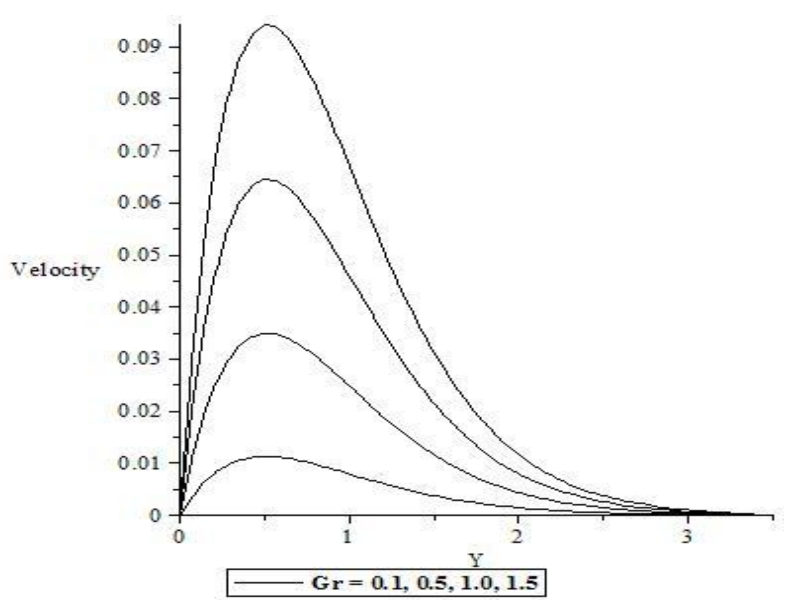

Figure 5: Variation of Velocity against y for different values of Gr.

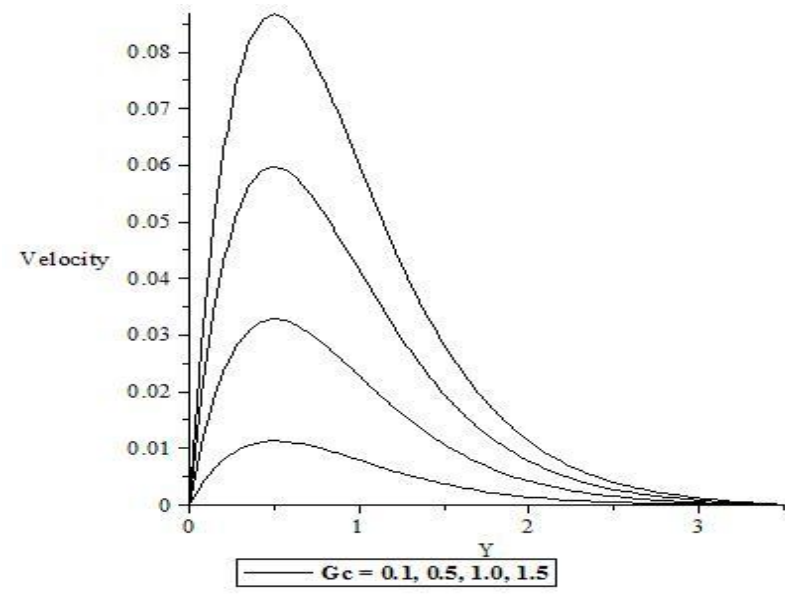

Figure 6: Variation of Velocity against y for different values of Gc.

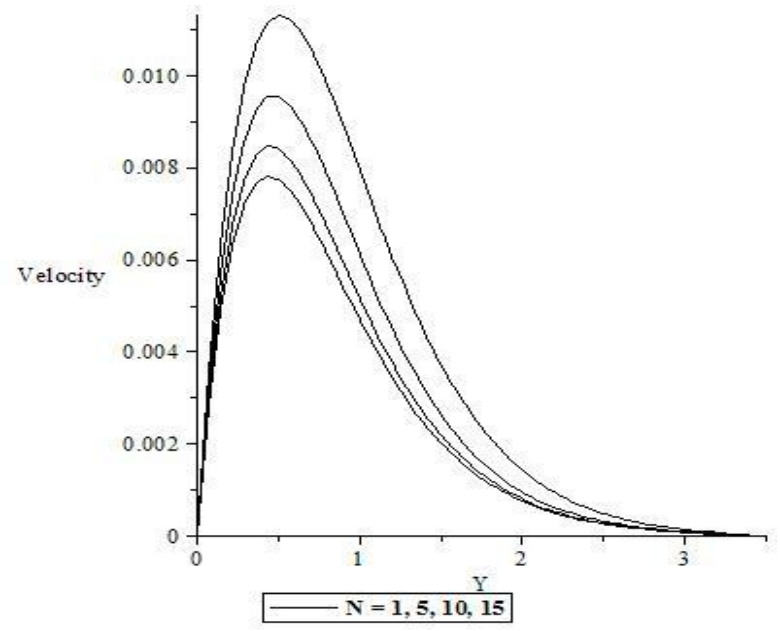

Figure 7: Variation of Velocity against y for different values of $\mathrm{N}$.

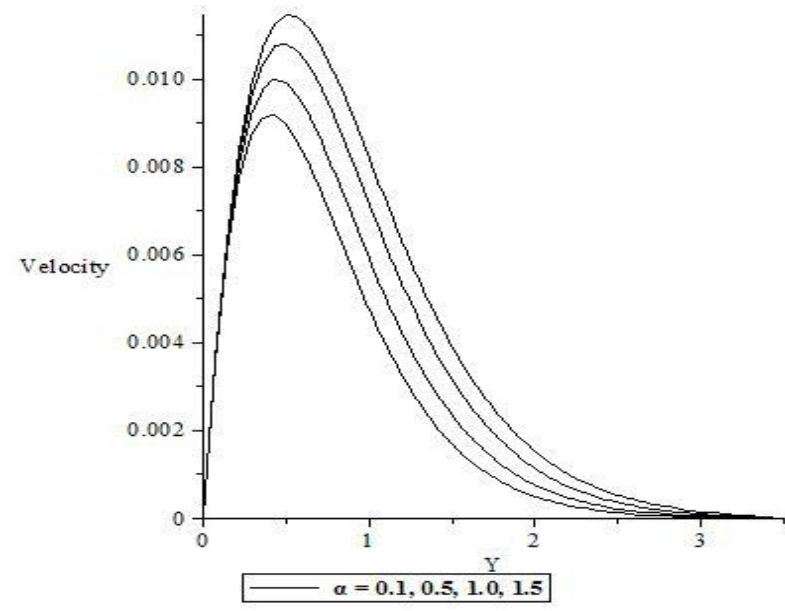


Figure 8: Variation of Velocity against y for different values of $\alpha$.

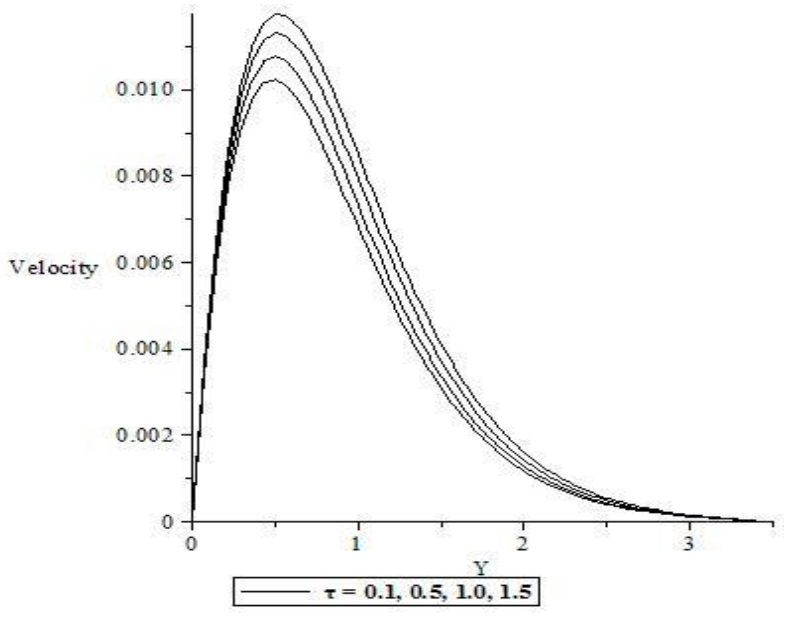

Figure 9: Variation of Velocity against y for different values of $\tau$.

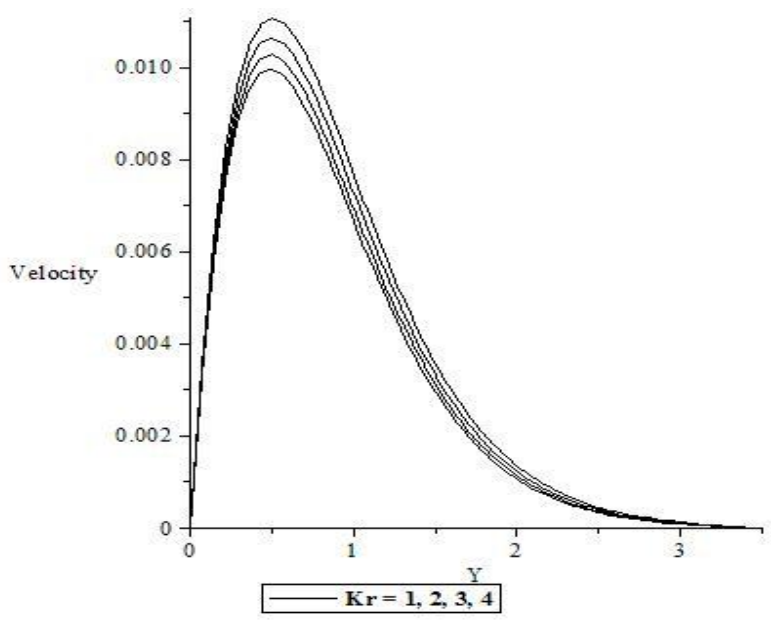

Figure 10: Variation of Velocity against y for different values of $\mathrm{Kr}$.

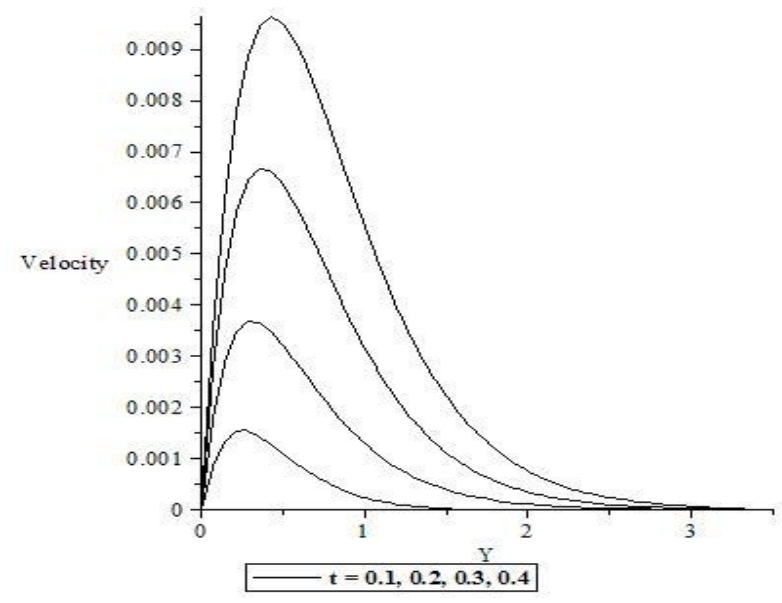

Figure 11: Variation of Velocity against y for different values of $\mathrm{t}$.

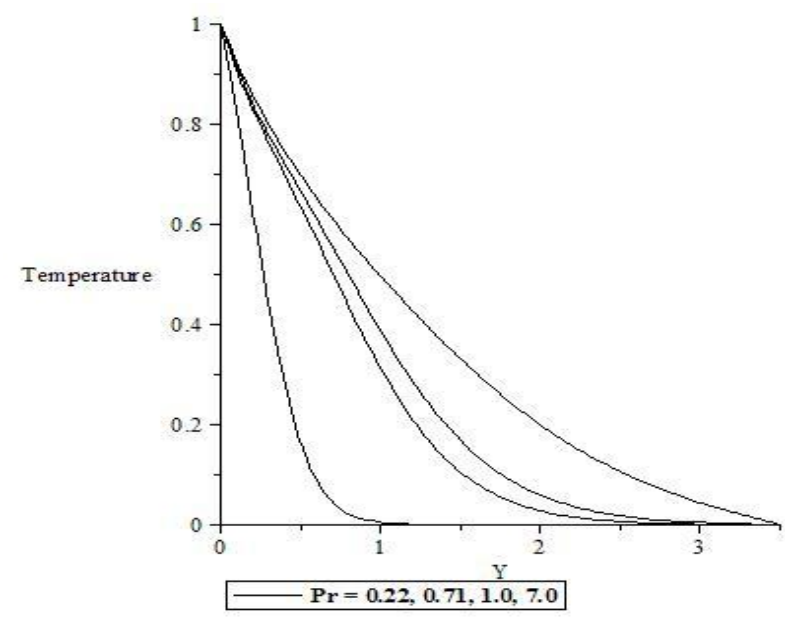

Figure 12: Variation of Temperature against y for different values of $\mathrm{Pr}$.

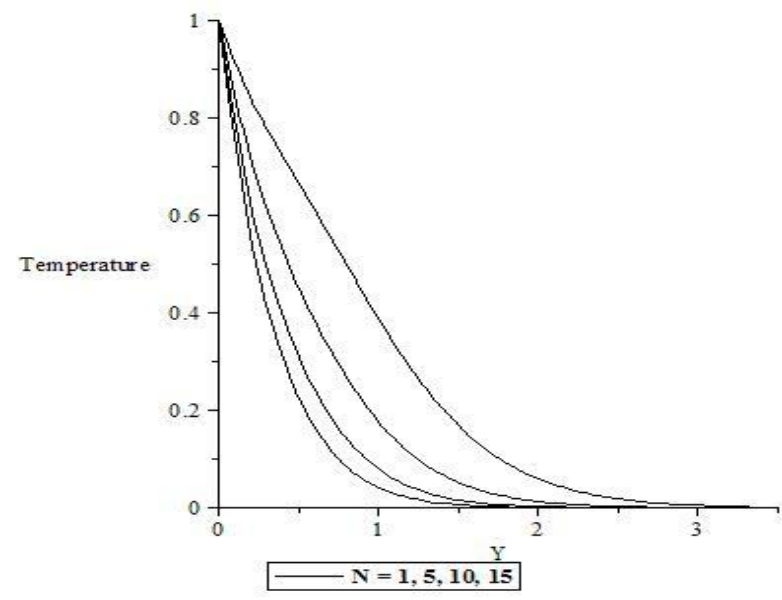

Figure 13: Variation of Temperature against y for different values of $\mathrm{N}$.

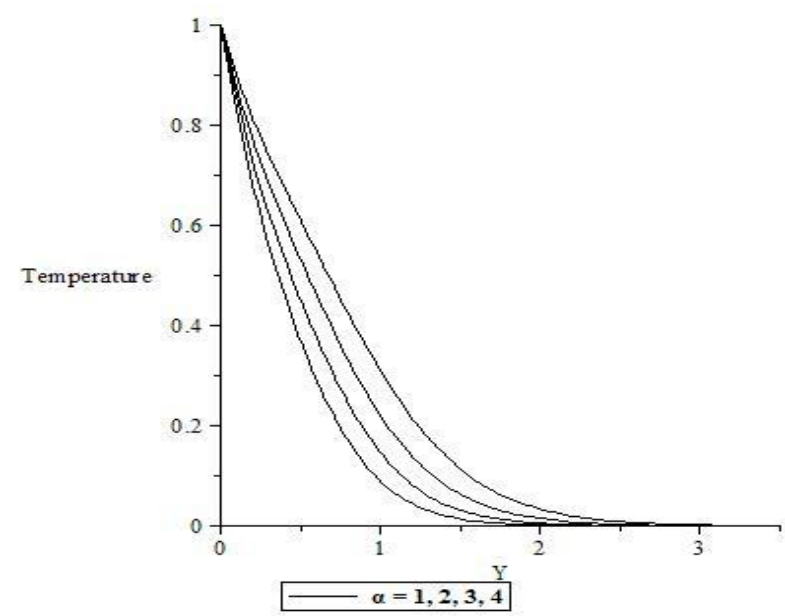


Figure 14: Variation of Temperature against y for different values of $\alpha$.

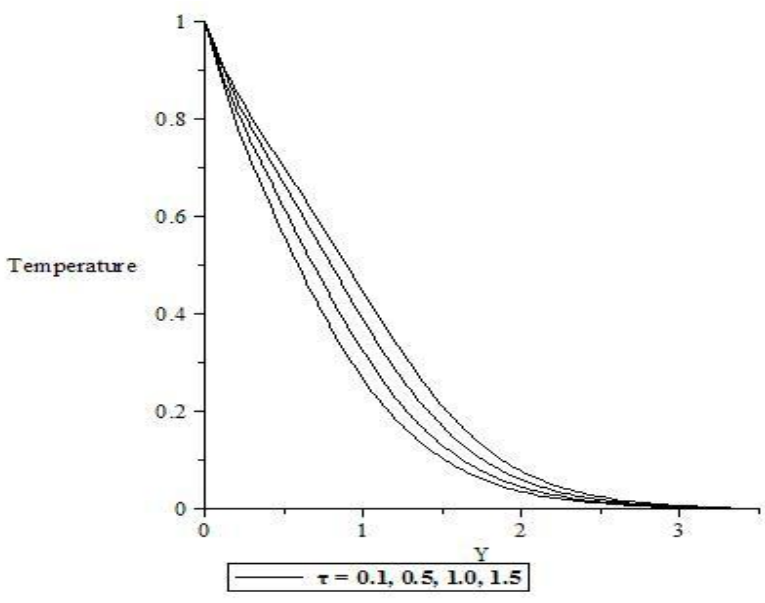

Figure 15: Variation of Temperature against y for different values of $\tau$.

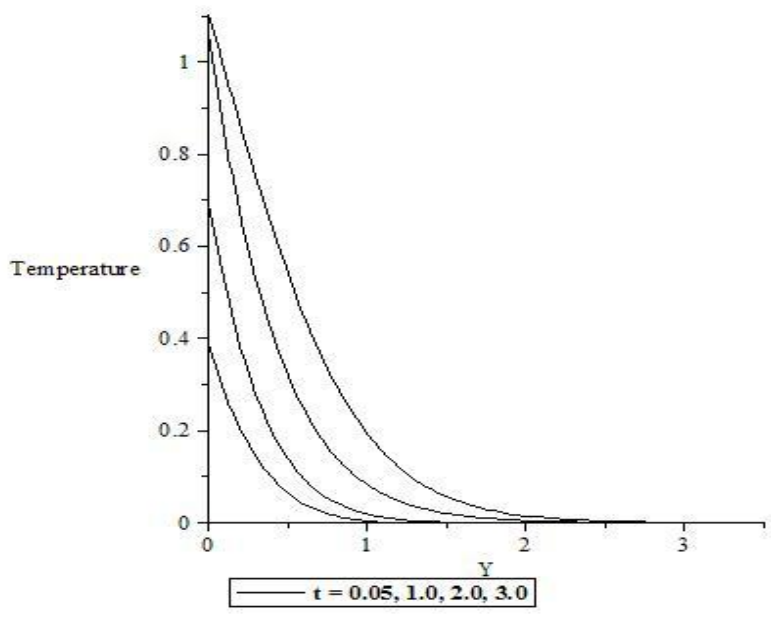

Figure 16: Variation of Temperature against $\mathrm{y}$ for different values of $\mathrm{t}$.

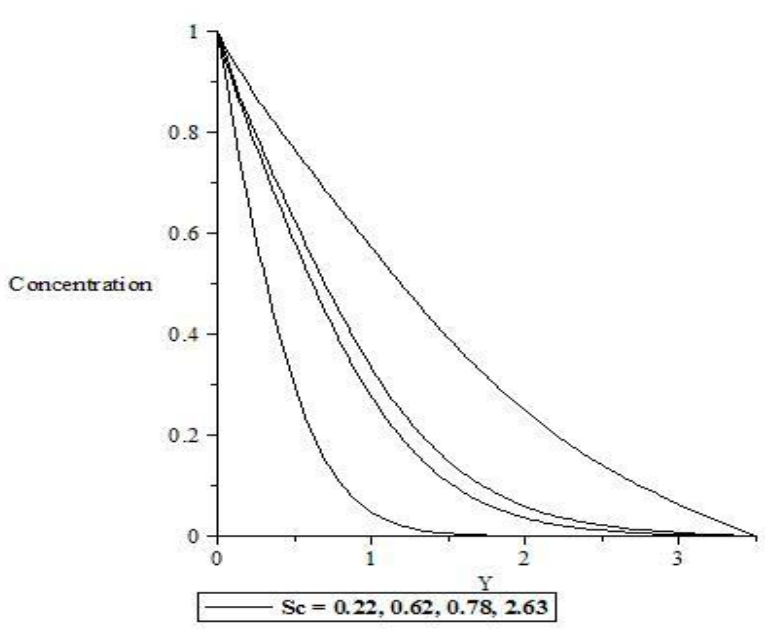

Figure 17: Variation of Concentration against y for different values of Sc.

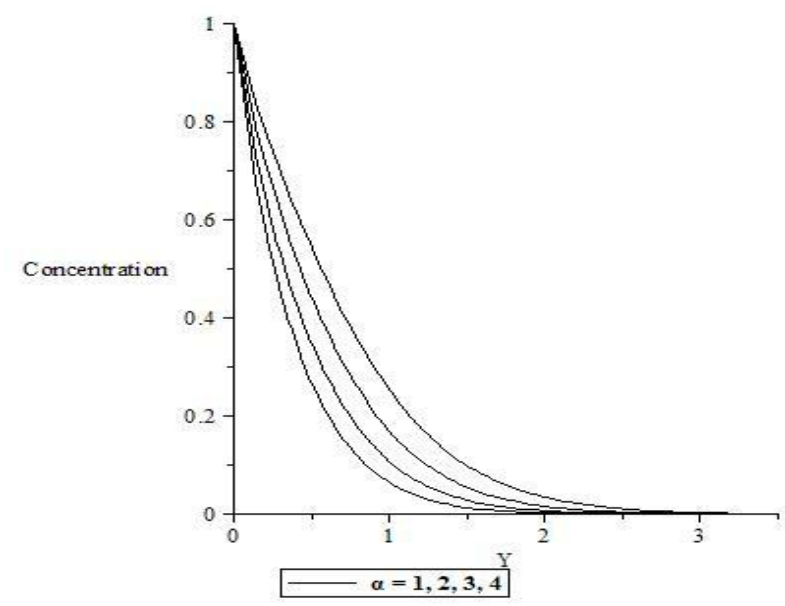

Figure 18: Variation of Concentration against y for different values of $\alpha$.

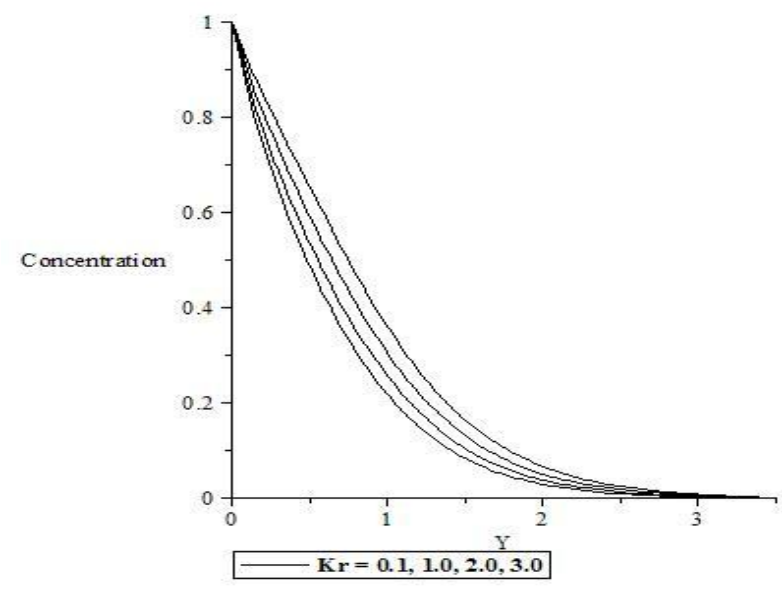

Figure 19: Variation of Concentration against y for different values of $\mathrm{Kr}$.

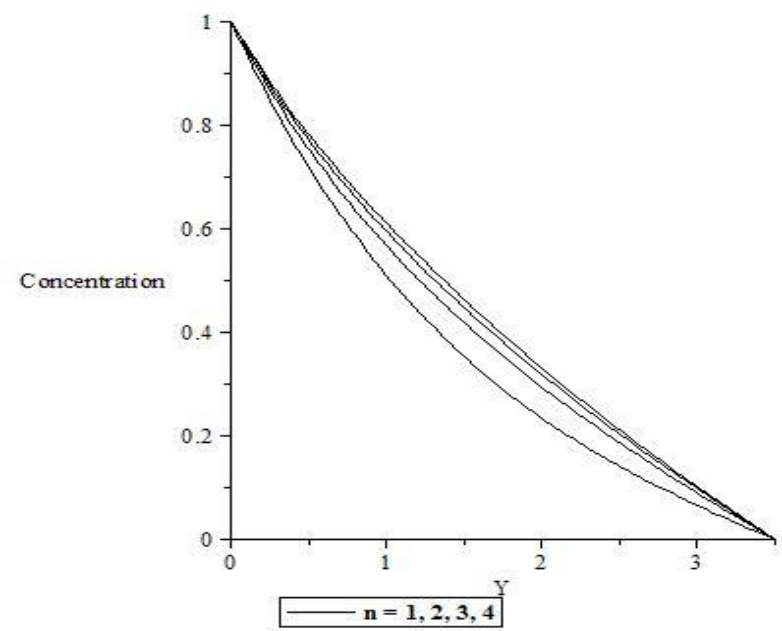


Figure 20: Variation of Concentration against y for different values of $n$.

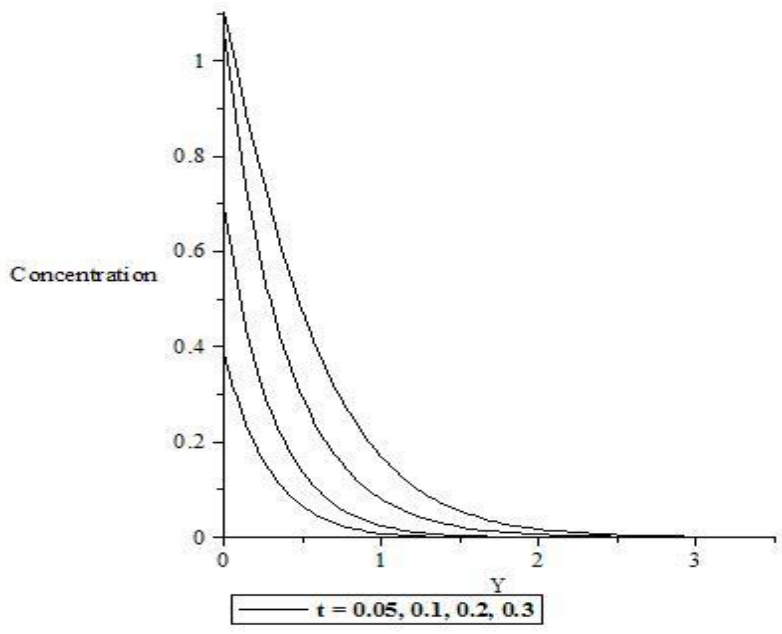

Figure 21: Variation of Concentration against $y$ for different values of $t$.

\section{CONCLUSIONS}

The paper studied the heat and mass transfer flow past an infinite vertical pate with variable thermal conductivity. The dimensionless governing equations are simplified, nondimensionalized and then solved numerically with the aid of MAPLE package. The effect of different parameters such as Prandtl number (Pr), Schmidt number (Sc), Eckert number $(\mathrm{Ec})$, magnetic field $(\mathrm{M})$, porosity $(\mathrm{K})$, thermal Grashof number $(\mathrm{Gr})$, mass Grashof number $(\mathrm{Gc})$, radiation $(\mathrm{N})$, suction $(\alpha)$, thermal Conductivity $(\tau)$, chemical reaction $(\mathrm{Kr})$ and reaction order (n) were studied. The conclusion shows that:

- The velocity increase with increase in the thermal Grashof number, mass Grashof number, thermal conductivity parameter, porosity parameter and time. Whereas the velocity decrease with increasing Prandtl number, Schmidt number, magnetic field parameter, radiation parameter, suction parameter and chemical reaction parameter.

- The temperature increase with increase in thermal conductivity parameter and time. But, the temperature decrease with increasing Prandtl number and suction parameter.

- The concentration increase with increasing reaction order parameter and time while the concentration decrease with increase in Schmidt number, suction parameter and chemical reaction parameter.

\section{REFERENCES}

[1] Muthucumarswamy, R. and Senthil K. 2004. Heat and mass transfer effects on moving vertical plate in the presence of thermal radiation. Theoretical Applied Mechanics, 31, 35-46.

[2] Gupta, P. S. and Gupta, A. S. 1977. Heat and mass transfer on a stretching sheet with suction. Canadian Journal of Chemical Engineering, 55, 744-746.

[3] Sattar, M. A. 1994. Free convection and mass transfer flow through a porous medium past an infinite vertical plate with time dependant temperature and concentration. International Journal of Pure and Applied Mathematics, 23, 759-766.

[4] Israel-Cookey, C. Ogulu, A. and Omubo-Pepple, V. B. 2003. Influence of viscous dissipation and radiation on unsteady MHD free-convection flow past an infinite heated vertical plate in a porous medium with timedependent suction. International Journal of Heat and Mass Transfer. 46, 2305-2311.

[5] Illingworth, C. R. 1950. Unsteady laminar flow of gas near an infinite plate. Proceeding of Cambridge Philosopical Society, 44, 603-613.

[6] Gebhart, B. 1962. Effects of viscous dissipation in natural convection. Journal of Fluid Mechanics, 14, 225-232.

[7] Soundalgekar,V. M., Pohanerkar, S. G. and Lahurikar, R. M. 1997. Transient free convection flow of an incompressible viscous dissipative fluid. Warme und Stoff, 32, 301-305.

[8] Das, U. N., Deka, R. K. and Soundalgekar, V. M. 1996. Radiation effects on flow past an impulsively started infinite isothermal plate. Journal of Theoretical Mechanics, 1, 111-115.

[9] Riley, K. F., Hobson, M. P. and Bence, S. J. 2006. Mathematical Methods for Physics and Engineers. Third Edition, Cambridge University Press, New York.

[10] Nield, D. A. and Bejan, A. 2006. Convection in Porous Media, 3rd Edition. Springer, New York.

[11] Kishan, N. and Srinivasmaripala, A. 2012. MHD mixed convection, heat and mass transfer on an isothermal vertical plate. Advances in Applied Science Research, 3(1), 60-74.

[12] Ramachandra, P. V. and Bhaskar, R. N. 2008. MHD free convection flow past a semi-infinite vertical permeable plate Indian Journal of Pure and Applied Physics, 46, 8192.

[13] Chamkha, A. J. (1997). Solar radiation assisted natural convection in a uniform porous medium supported by a vertical flat plate. ASME, Journal of Heat Transfer, 119, 89-96.

[14] Sangapatnam, S., Nandanoor, R., and Vallampati, R. P. 2009. Radiation and mass transfer effects on MHD free convection flow past impulsively-started isothermal vertical plate with dissipation. Thermal Science, 13(2), 171-181.

[15] Yasar, O. and Moses, G. A. 1992. R-MHD: An Adaptivegrid radiation magnetohydrodynamic Computer code. Computer Physics Communications, 69(2-3), 439-458.

[16] Aboeldahab, E. M. and El-Din, A. G. 2005. Thermal radiation effects on MHD flow past a semi-infinite inclined plate in the presence of mass diffusion. Heat and Mass Transfer. 41(12), 1056-1065.

[17] Alagoa, K. D. Tay, G. and Abbey, T. M. 1998. Radiative and free convective effects of MHD flow through a porous medium between infinite parallel plates with time-dependent suction. Astrophysics and Space Science. 260(4), 455-468. 
[18] Nield, D. A. and Bejan, A. 1999. Convection in Porous Media, 2nd Edition. Springer, New York.

[19] Bejan, A. and Kraus, A. D. 2003. Heat Transfer Handbook, Wiley, New York.

[20] Ingham, D. B., Bejan, A., Mamut, E. and Pop, I. 2004. Emerging Technologies and Techniques in Porous Media. Kluwer, Dordrecht.
[21] Suneetha, S., Bhaskar, R. N. and Ramachandra, P. V. 2009. Radiation and mass transfer effects on MHD free convection flow past an impulsively started isothermal vertical plate with dissipation. Thermal Science, 13(2), $71-181$.

[22] Muthucumaraswamy, R. and Meenakshisundaram, S. 2006. Theoretical study of chemical reaction effects on vertical oscillating plate with variable temperature. Theoretical Applied Mechanics, 33(3), 245-257. 